\title{
Wie Gerechtigkeit in die Soziale Arbeit kommt
}

\section{Als Experten für soziale Probleme und deren Bearbeitung die Ethik der Sozialen Arbeit beeinflussen}

\author{
Matthias Möhring-Hesse
}

Prof. Dr. Matthias Möhring-Hesse ist Hochschullehrer für philosophische und theologische Grundlagen des sozialen Handelns an der Hochschule Vechta. E-Mail matthias.moehring-hesse@uni-vechta.de
Die Soziale Arbeit ist nicht die Autorin ihrer eigenen Ethik. Mit ibrer öffentlichen Beauftragung werden ibr auch die normativen Ziele vorgegeben. Was ibr dagegen an noch so edlen Zielen nicht vorgegeben wird, wird sie selbst nachträglich nicht einziehen können. Jedoch können sich Sozialarbeiterinnen und Sozialarbeiter in den öffentlichen Debatten einmischen, in denen über die normativen Ziele der Sozialen Arbeit entschieden wird. Und sie sollten dies tun-auch im Namen ibrer Klientinnen und Klienten.

In der Welt der Witze konnten sich Sozialarbeiterinnen und Sozialarbeiter etablieren, wenngleich mit deutlichem Abstand zu Ostfriesen und Blondinen. In einigen Witzen gelten sie als besonders faul, in anderen als pseudo-professionell mit Hang zum sozialwissenschaftlichen Slang - und in wieder anderen als ungemein gutwillig und "sozial ", wobei sie daraus kein Geheimnis machen. Zumindest das sich in den zuletzt genannten Witzen ausdrückende Vorurteil wird in einer von der International Federation auf Social Workers (IFSW) vorgeschlagenen »internationalen Definition der Profession Soziale Arbeit « eindrucksvoll bestätigt: »Soziale Arbeit ist eine Profession, die sozialen Wandel, Problemlösungen in menschlichen Beziehungen sowie die Ermächtigung und Befreiung von Menschen fördert, um ihr Wohlbefinden zu verbessern. ... Dabei sind die Prinzipien der Menschenrechte und sozialer Gerechtigkeit für die Soziale Arbeit von fundamentaler Bedeutung. «

Hehre Ansprüche werden damit für die Soziale Arbeit formuliert, mehr noch: ihr zur Grundlage gemacht, so dass ohne eine Aufklärung dieser normativ gehaltvollen Ansprüche, mithin ohne Ethik gar nicht verstanden werden kann, was Soziale Arbeit »ist «. Nimmt man diese »internationale Definition « als Grundlage für das Verständnis von Sozialer Arbeit, dann darf man sich fragen, wie denn diese normativ gehaltvollen Ansprüche überhaupt »in « die Soziale Arbeit kommen und dort zu Grundlagen wurden.

Diese Frage findet in der einschlägigen Literatur zumeist eine professionsbezogene Antwort: Die Profession selbst gibt sich - in Anlehnung an internationale Deklarationen etwa der Menschenrechte oder in Referenz auf Großtheorien der philosophischen Ethik - ihre eigene Ethik und vertritt diese gegenüber den Klienten und deren Anspruch auf »Hilfe « sowie gegenüber dem öffentlichen Auftraggeber, zumeist staatlichen Institutionen und deren Anspruch auf »Kontrolle«. Gleichsam aus eigenem Antrieb bestimmt sich Soziale Arbeit dann auf der Grundlage von sozialer Gerechtigkeit und verpflichtet sich, Gerechtigkeit im Lande und manchmal auch über dessen Grenzen hinweg zu mehren.

Sonderlich belastbar ist die Annahme nicht, die Soziale Arbeit sei selbst die Autorin ihrer eigenen Ethik. Selbstverständlich wird man sich im Kreise derer, die Soziale Arbeit als ihren Beruf haben, auf gemeinsame Prinzipien verständigen müssen und können, wie man diese Arbeit mit welchen Zielen ausüben soll. Doch grundlegend ist Soziale Arbeit normativ fremd, nämlich durch ihre öffentlichen Auftraggeber bestimmt. Ihre normativen Ziele werden ihr vorgegeben. So ist für die Soziale Arbeit auch vorentschieden, ob und was sie zur Gerechtigkeit in einer Gesellschaft beitragen soll.

\section{Außerhalb der Sozialen Arbeit}

Über ihre unterschiedlichen Tätigkeiten hinweg dürfte es ein gemeinsamer Anspruch von Sozialarbeitern sein, dass sie ihre Arbeit gut machen. Für sie heißt das vor allem, dass sie in ihrer Beratung, Betreuung, Unterstützung und Begleitung den jeweiligen professionellen Standards 
genügen. Was diese Standards sind, klären sie, unterstützt durch die auf ihre Arbeit bezogenen Wissenschaften, mit ihren Kolleginnen und Kollegen, idealerweise mit der »community « aller ihrer Kolleginnen und Kollegen.

In diesem Umfeld erweist sich die Professionalität, so eine weit verbreitete Überzeugung, in einem Spannungsverhältnis zwischen allgemeiner Wissensapplikation und Fallverstehen, wobei Theorie und Praxis wechselseitig aufeinander bezogen und daher in Sozialer Arbeit wissenschaftliches Wissen gerade nicht »angewandt " wird. Allerdings können die Sozialarbeiter ihre Professionalität nicht einfach unter sich ausmachen und untereinander entsprechende Standards vereinbaren. Ob sie im Einzelfall professionelle Arbeit leisten, wird wie die Frage, was überhaupt die Professionalität ihrer Arbeit ausmacht, auch von ihren Klienten, von ihren Auftraggebern und schließlich von einer interessierten Öffentlichkeit beurteilt, die sich in ihren Urteilen durchaus von eigenständigen und deswegen abweichenden Vorstellungen leiten lassen können.

In der erwähnten »internationalen Definition « wird das »Prinzip der Sozialen Gerechtigkeit « mit zu den Grundlagen der Sozialen Arbeit genommen und so als einer der Standards professioneller Sozialer Arbeit geführt. Der damit erhobene Anspruch kann sich einerseits auf das Angebot beziehen, sofern die Möglichkeit, in bestimmten Problemfällen auf die Beratung, Begleitung, Unterstützung und Betreuung durch Professionelle zurückgreifen zu können, die Gerechtigkeit in einer Gesellschaft mehrt. Andererseits kann er sich aber auch auf die Soziale Arbeit im konkreten Fall beziehen, indem die Klienten durch professionelle Beratung, Begleitung, Unterstützung und Betreuung in eine nach Maßgabe der Gerechtigkeit gewünschte Lage versetzt werden.

In beiden Fällen wird mit »Gerechtigkeit « eine Wirkung der Sozialen Arbeit außerhalb ihrer selbst und zwar auch außerhalb der für Soziale Arbeit typischen, wenn nicht gar konstitutiven Beziehungen der Koproduktion von Klienten und Professionellen intendiert. Damit in Übereinstimmung referieren wir zumindest alltagssprachlich mit » Gerechtigkeit « auf die grundlegende Ordnung von sozialen Beziehungen oder - um mit John Rawls zu sprechen - auf die »Grund- struktur « einer Gesellschaft. Wird nun Gerechtigkeit zur Grundlage der Sozialen Arbeit "gemacht ", wird sie im Sinne dieser alltagssprachlichen Begriffsverwendung in der jeweils referierten Gesellschaft eingeordnet und von deren "Grundstruktur" her beurteilt. Ob die Soziale Arbeit zur Mehrung von Gerechtigkeit beiträgt, entscheidet sich daher nicht in der Beziehung zwischen Sozialarbeitern und ihren Klienten, sondern in Referenz auf die Gesellschaft und deren grundlegende Ordnung.

\section{Soziale Gerechtigkeit durch Soziale Arbeit}

Zumeist werden in dem mit "Soziale Arbeit« bezeichneten Tätigkeiten Menschen in ihrem Bemühen um »Lebensbewältigung « unterstützt - und zwar dadurch, dass Professionelle geeignete Ressourcen, Räumen und Gelegenheiten organisieren. Auf derartige Unterstützung sind Menschen zu allen Zeiten und an allen Orten angewiesen, aber sie können sich in modernen Gesellschaften dieser Unterstützung immer weniger privat sichern. Zudem sind die Ansprüche und Erwartungen an derartige Unterstützung derart gewachsen, dass sie nicht einfach nur in guter Absicht und mit gemeinem Menschenverstand gegeben werden kann, sondern eine entsprechende Qualifizierung, eine berufliche und darüber kontinuierliche sowie eine reflektierte Ausübung, mithin ihrer Professionalisierung bedarf.

Professionelle Unterstützung zur »Lebensbewältigung « ist für die Lebensverhältnisse aller Menschen und für ihre Chancen, der Gesellschaft mit allen anderen und in Augenhöhe zu allen anderen anzugehören, ähnlich konditional geworden, wie Rechte und Freiheiten oder wie der Anteil am gemeinsam erwirtschafteten Reichtum. Somit betrifft der Zugang $\mathrm{zu}$ professionellen Leistungen der Beratung, Begleitung, Unterstützung und Betreuung tatsächlich die Grundstruktur einer Gesellschaft - und ist der Zugang zu diesen Diensten daher eine Frage der sozialen Gerechtigkeit.

Auch in einer zweiten Hinsicht kann der Beitrag der Sozialen Arbeit zur sozialen Gerechtigkeit bestimmt werden: Mit John Rawls haben in einer gerechten Gesellschaft alle zumindest die gleichen grundlegenden Freiheiten und Rechte; zudem besteht faire Chancengleichheit, unterschiedliche soziale Positionen einnehmen und Ämter besetzen zu können. Diese beiden Eigenschaften einer gerechten Gesellschaft realisieren sich nicht durch einfaches Dekret, etwa durch die Setzung gleicher Rechte. In vielen Fällen ist eine professionelle Unterstützung Voraussetzung dafür, dass Menschen die ihnen zustehenden Freiheiten und Rechte nicht nur formal »haben ", sondern auch tatsächlich wahrnehmen können. Professionelle Unterstützung ist auch Bedingung dafür, dass sie ihre vergleichsweise schlechteren Voraussetzungen ausgleichen können und dadurch gleiche Chancen wie alle anderen haben. Soziale Arbeit, die die Wahrnehmung von Freiheiten und Rechte ermöglicht und gleiche Chancen eröffnet, kann als Bedingung dafür ausgewiesen werden, dass die als gerecht beurteilte Grundstruktur tatsächlich besteht und in diesem Sinne die Gesellschaft gerecht ist.

\section{Pflicht zur Gerechtigkeit}

Sozialarbeiter arbeiten in konkreten »Fällen « - und haben es dabei mit Menschen mit Problemen und daraus erwachsenden, manchmal nur vermuteten Bedarfen an Unterstützung zu tun. Dass sie auf diese Bedarfe reagieren (dürfen), erwächst aber weder unmittelbar aus den Bedarfen ihrer Klienten, noch aus ihrer eigenen Willkür, für bestimmte Probleme bestimmter Menschen zuständig zu sein, oder aus der Ethik ihres Berufes, auf bestimmte Bedarfe von Menschen reagieren $\mathrm{zu}$ sollen. Auf welche Bedarfe sie reagieren dürfen, das ist - in welchen Vermittlungen auch immer - durch die öffentlichen Auftraggeber der Sozialen Arbeit und damit in der Bundesrepublik zumeist durch den Sozialstaat vorentschieden.

Der Sozialstaat erkennt Probleme einzelner Menschen oder bestimmter Gruppen als soziale Probleme an, die dadurch zwar immer noch an den einzelnen Menschen oder an den jeweiligen Gruppen hängen, aber nicht mehr nur deren Probleme sind. Als Folge des Anerkennens von sozialen Problemen bietet der Staat (oder andere gesellschaftliche Akteure) denjenigen, die diese Probleme »in erster Person « haben, Unterstützung durch Professionelle an. Mit der tatsächlichen Er- 
stellung entsprechender Unterstützungsleistung beauftragt der Staat zumeist nichtstaatliche Einrichtungen - und stattet sie mehr oder weniger hinreichend mit den notwendigen finanziellen Mitteln aus. Diese wiederum beschäftigen Sozialarbeiter mit der professionellen Beratung, Begleitung, Unterstützung und Betreuung von Menschen oder Gruppen mit entsprechenden Problemen. Den Professionellen ist aber Wesentliches ihrer Sozialen Arbeit vorgegeben, vor allem sind die auslösenden Bedarfe typisierend bestimmt, wie auch die Konditionen, unter denen bedürftige Menschen Unterstützung in Anspruch nehmen können. Vorgegeben sind auch die Leistungen, mit denen sie typischerweise auf typische Bedarfe, dabei mit welchen Zielen, mit welchem Aufwand und mit welchen Mitteln reagieren können.

Wenn nun der angeführte Gerechtigkeitsbezug der Sozialen Arbeit besteht, dann muss er bereits »in « diesen Vorgaben »stecken «. Soziale Probleme und die zur ihrer Bearbeitung vorgesehenen Unterstützungsleistungen müssen entsprechend bestimmt werden, dass einzelnen oder Gruppen die Unterstützung zur »Lebensbewältigung « erhalten, dass sie ihr Leben in einer Gesellschaft mit allen anderen und auf Augenhöhe zu diesen führen können. Dass Soziale Arbeit zur Gewährleistung gleicher Freiheiten und Rechte sowie zur Verwirklichung fairer Chancengleichheit beiträgt, muss ebenfalls bei der Auswahl der Probleme, die Soziale Arbeit "auslösen ", und der Beauftragung von Unterstützungsleistung vorgesehen werden. Wird dagegen Soziale Arbeit ohne diese Gerechtigkeitsbezüge in Auftrag gegeben, wird sie diese selbst nicht nachträglich einziehen können. Trotz aller Spielräume, die Sozialarbeiter bei der Erbringung von Leistungen in der Beziehung zu ihren Klienten haben, sie haben weder als Einzelne noch über ihre Profession die Definitionsmacht über die sozialen Probleme, die Soziale Arbeit auslösen können, oder über die Ziele, mit denen Soziale Arbeit als Lösung von sozialen Problemen vorgesehen wird.

Wenn überhaupt, wird in der einschlägigen Literatur die öffentliche Beauftragung der Sozialen Arbeit mit Begriffen wie "Kontrolle « oder "Normalisierung « angesprochen. Mit diesen und ähnlichen Begriffen hält man die Soziale Arbeit zumindest auf der Ebene der Theorie - in
Distanz zu ihren öffentlichen Auftraggebern und erlaubt ihr so, sich kritisch auf deren Auftrag zu beziehen. Zugleich besteht aber die Gefahr, sich in dieser Distanz zu verfangen und den mit Begriffen wie "Kontrolle" und "Normalisierung « ausgewiesenen Auftrag ethisch zu neutralisieren. Dieser Gefahr erliegt man insbesondere dann, wenn man die Ethik der Sozialen Arbeit, allen voran ihren Gerechtigkeitsbezug, über die eigene Profession und damit an der öffentlichen Beauftragung vorbei zu begründen sucht.

Dass in der - mit welchen Begriffen auch immer rekonstruierten - öffent-
Die Bedeutung der Sozialen Arbeit für die soziale Gerechtigkeit entscheidet sich letztlich in diesen öffentlichen Debatten und damit außerhalb der Sozialen Arbeit selbst. Allerdings ist dieser Ort für die Professionellen nicht unzugänglich, sondern steht ihnen - im Gegenteil - offen. Als Experten für soziale Probleme und für die professionelle Unterstützung der davon »in erster Person « Betroffenen können sie sich an entsprechenden Meinungsund Willensbildungsprozessen und darüber an der normativen Programmierung der Sozialen Arbeit mitwirken. Das ist dann weniger die Aufgabe einzelner Sozi-

\section{"Soziale Arbeit kann nicht selbst definieren, wann sie tätig werden}

\section{muss «}

lichen Beauftragung normativ gehaltvolle Ziele stecken, sollte theoretisch leichter zugestanden werden können, so diese nicht unmittelbar den direkten Auftraggebern, schon gar nicht dem Staat, sondern der sie kontrollierenden Öffentlichkeit zugerechnet werden müssen. Die öffentlichen Auftraggeber von Sozialer Arbeit und unter ihnen vor allem der Sozialstaat sind an öffentliche Meinungsund Willensbildungsprozesse rückgebunden, in denen sich zumindest auf Dauer bewähren muss, dass bestimmte Probleme - im Gegensatz zu anderen Problemen - als soziale Probleme anerkannt werden, dass für diese Probleme öffentliche Unterstützung bereitgestellt und diese mit Zielvorgaben Professionellen zur Aufgabe gemacht wird. Spätestens in diesen Meinungs- und Willensbildungsprozessen werden entsprechende Vorgaben der Sozialen Arbeit vor dem Hintergrund von Gerechtigkeitsvorstellungen besprochen; so wird geprüft, ob sie gesellschaftsweit und zumindest in diesem Sinne allgemein gerechtfertigt werden können. Dabei kann die auf den Sozialstaat bezogene Öffentlichkeit durchaus initiativ werden, indem sie dem Sozialstaat die Wahrnehmung bestimmter Probleme als soziale Probleme aufdrängen und die Gewährleistungen entsprechender Dienste unter bestimmten Bedingungen und Auflagen abverlangen kann. alarbeiter, als vielmehr deren gemeinsame Aufgabe, die sie über ihre Berufsverbände, aber auch über ihre Einrichtungen und deren Träger, etwa die Wohlfahrtsverbände, leisten können. Entsprechend organisiert - und durch die auf ihre Arbeit bezogenen Wissenschaften ausreichend munitioniert - bieten sie in den öffentlichen Aushandlungsprozessen professionell gestützte Argumente dafür, soziale Probleme zu sehen und mit öffentlich verantworteten Unterstützungsleistungen zu beantworten.

Dabei müssen sie nicht nur ihre eigenen Interessen als Beruf, als Einrichtungen und als Träger vertreten, sondern sollten zugleich auch die Interessen ihrer Klienten advokatorisch vertreten. Denn als Experten für soziale Probleme und deren professionelle »Bearbeitung « erweisen sich Sozialarbeiterinnen und Sozialarbeiter in der Öffentlichkeit erst in dem Maße, als sie als glaubwürdige Anwälte derer auftreten können, die im Vollzug der Sozialen Arbeit ihre Klienten sind oder sein sollen. Indem sie dieses politische Mandat außerhalb von Sozialer Arbeit annehmen, wirken sie daran mit, dass ihre Soziale Arbeit tatsächlich mit normativ gehaltvollen Zielen beauftragt und ihr soziale Gerechtigkeit so zur Grundlage gemacht wird, wie es in der »internationalen Definition « zurecht intendiert wird. 


\section{Was versteht man eigentlich unter »sozialer Gerechtigkeit«?}

Soziale Gerechtigkeit ist eine geradezu konstitutive Kategorie der Wissenschaft und Praxis der Sozialpolitik. Soziale Rechtfertigungsdiskurse und politische Ordnungsvorstellungen standen religionsgeschichtlich bereits am Anfang menschlicher Kulturentwicklung. Geschlechterordnungen waren implizit ebenso ein Thema wie die kulturelle Umgangsweise mit vulnerablen Gruppen (alte Menschen, Witwen und Waisen etc.).

Rechtsphilosophisch muss soziale Gerechtigkeit als die zentrale Idee des Rechts überhaupt verstanden werden. Das Verhältnis zu Solidarnormen ist allerdings kontrovers: Soziale Gerechtigkeit kann Solidarität nach sich ziehen, muss es aber nicht. Geklärt werden muss: Solidarität mit wem, durch wen und warum? Die Schlüsselfrage einer jeden Sozialpolitik ist: Wer bekommt was, wie und warum?

Zum näheren systematischen Verständnis von sozialer Gerechtigkeit ist zwischen der distributiven und der redistributiven Gerechtigkeit zu unterscheiden. In Bezug auf moderne Marktgesellschaften ist unter redistributiver Gerechtigkeit ein Prinzip der Umverteilung als Korrektur der primären Marktverteilung zu verstehen. Unter distributiver Gerechtigkeit ist die lebenslaufbezogene Chancengleichheit als Chancenverteilung (Bildung, Gesundheit etc.) zu verstehen. Beide Formen können theoretisch unterschieden werden, sie gehen in der geschichtlich-gesellschaftlichen Realität jedoch Synthesen ein.

Rechtsphilosophisch fundamental für eine optimale Mischung ist das Grundrecht auf freie Entfaltung der menschlichen Persönlichkeit ( $\mathbb{1}$ SGB I im Lichte des Art. 2 GG). Dieses verweist aus der Perspektive einer angemessenen philosophischen Anthropologie der menschlichen Existenz auf die Begrenzung der Freiheit des einzelnen Bürgers durch das gleiche Grundrecht der anderen Bürger: Person-Sein ist nur im Modus des sozialen Mitseins möglich. Das System der Sozialgesetzbücher materialisiert somit aus der Perspektive des Sozialstaatsgebotes des Art. 20 GG dieses Grundrecht der Person. Soziale Gerechtigkeit nimmt im Lichte empirischer Befunde im Verständnis der Bürger aber sektorspezifische Formen an: z. B. Bedarfsgerechtigkeit in der Gesetzlichen Krankenversicherung und Äquivalenzgerechtigkeit in der Gesetzlichen Rentenversicherung. Der Sozialstaat weist entsprechend vielfältige Mischungen von unterschiedlichen Gerechtigkeitskonzepten auf, je nach sozialem Risiko und Verständnis von Kausalitäten: Eigenverantwortung vs. soziales Schicksal, Verhaltensfreiheit vs. Determinismus etc. Offensichtlich wird das Verhältnis von Selbstständigkeit und Selbstverantwortlichkeit, von sozialer Mitverantwortung und sozialer Ressourcenabhängigkeit problemorientiert sehr unterschiedlich akzentuiert.

Das Prinzip der Subsidiarität fungiert hier als produktive Leerformel und muss im sozialen Raum und in der historischen Zeit immer wieder neu problemorientiert gefüllt und politisch gelebt werden. Schutzrechte des Bürgers und Förderrechte stellen komplementäre Größen dar; die ältere verfassungsrechtliche Position der Unvereinbarkeit von Rechts- und Sozialstaatlichkeit ist heute nicht mehr haltbar. Wichtig bleibt aber die Einsicht, dass nur der Rechtsstaat, nicht aber der Sozialstaat der Akteur ist.
Zunehmende Bedeutung (auch europarechtlich) gewinnt ein dreidimensionales Verständnis des Menschen als Bürger: als Wirtschafts-, Staats- und Sozialbürger. In das Zentrum rückt damit die Dogmatik der grundrechtlich verbürgten Rechte auf Teilhabe an den ökonomischen, politischen, sozialen und kulturellen Gütern. Sozialpolitisch konkretisiert sich die angeführte Materialisierung des Sozialstaatsprinzips durch Interventionen, die die personengebundenen Kompetenzen ebenso fördern und steigern wie die Sicherstellung von Umwelten (soziale Infrastrukturen, Daseinsvorsorge) der gelingenden Daseinsbewältigung der Menschen im Lebenslauf. Die hierbei angetriebenen » Modernisierungen « der Dienstleistungsmärkte hin zu mehr Markt- und Wettbewerbsorientierung betreffen effizienzorientiert die Art und Weise der Leistungserstellung, nicht aber das Grundrecht auf freien Zugang zu den »Dienstleistungen von allgemeinem (wirtschaftlichen) Interesse $«$.

Zum Gerechtigkeitsverständnis aktueller Diskurse gehört aber auch zunehmend das ökonomische Argument, dass die normativ begründeten sozialen Sicherungssysteme anreizkompatibel ausgestaltet sein, also Fehlverhalten minimieren sollen. Die moderne ökonomische Theorie sieht in diesem Lichte produktive Schnittflächen zwischen soziale Gerechtigkeit und Effizienz dort, wo Win-win-Situationen (nach dem sogenannten Pareto-Prinzip) realisiert werden können. Hier werden v. a. Humankapitalinvestitionen verstärkt eingefordert, um die Integrationsmöglichkeiten in die Arbeitsmärkte zu steigern (»Workfare « statt »Welfare«). Diese Effizienzstrategie wird durchaus als spezifisches Verständnis soziale Gerechtigkeit vorgetragen; sie verweist kulturgeschichtlich auf puritanisch-autoritäre Wurzeln sozialen Paternalismus. Immerhin kann die moderne ökonomische Theorie in Grenzen aufzeigen, wie sich Spielräume rationalen Altruismus abzeichnen: Da der Wohlstand der Bürger interdependent ist, sind Umverteilungsprozesse in bestimmten Grenzen im Konsens möglich.

Die moderne Psychologie konnte allerdings die Voraussetzungen dieser Umverteilungsbereitschaft aufdecken: Die Menschen müssen durch Sozialisation und Erziehung die Kompetenz zur Empathie erwerben, um selbst bindungs- und liebesfähig zu sein und auf dieser Basis auch für Mitbürger prosoziale Einstellungen entwickeln zu können. Die experimentelle Spieltheorie (Spiel/Spieltheorie) konnte zeigen, dass die Mehrzahl der Menschen nicht rein eigensinniges Verhalten zeigt, sondern auch faire Orientierungen einbringt und erwartet. Ob diese Fairness-Bereitschaft sowie die Neigung zum rationalen Altruismus ausreichen, um ein sozial gerecht geordnetes Zusammenleben zu ermöglichen, bleibt kontrovers. Oftmals muss auch eine Solidarität (einseitiges Gabeverhalten) möglich sein, die letztendlich nur als Barmherzigkeit und Gnade verstehbar bleibt.

Frank Schulz-Nieswandt

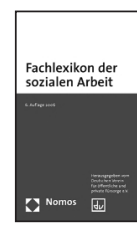

Quelle: Deutscher Verein für öffentliche und private Fürsorge e. V. (Hg.): Fachlexikon der sozialen Arbeit. 6. Auflage. Nomos Verlagsgesellschaft, Baden-Baden 2007. 1.195 Seiten. 44,- Euro. ISBN 978-3-83291825-5. Seite $851 \mathrm{f}$. 